\title{
Stimulating the Brain's Language Network: Syntactic Ambiguity Resolution after TMS to the Inferior Frontal Gyrus and Middle Temporal Gyrus
}

\author{
Daniel J. Acheson ${ }^{1}$ and Peter Hagoort ${ }^{1,2}$
}

\begin{abstract}
The posterior middle temporal gyrus (MTG) and inferior frontal gyrus (IFG) are two critical nodes of the brain's language network. Previous neuroimaging evidence has supported a dissociation in language comprehension in which parts of the MTG are involved in the retrieval of lexical syntactic information and the IFG in unification operations that maintain, select, and integrate multiple sources of information over time. In the present investigation, we tested for causal evidence of this dissociation by modulating activity in IFG and MTG using an offline TMS procedure: continuous theta-burst stimulation. Lexical-syntactic retrieval was manipulated by using sentences with and without a temporarily word-class (noun/verb) ambiguity (e.g., run). In one group of participants, TMS was applied to the IFG and MTG, and in a control group, no TMS was applied. Eye
\end{abstract}

\section{INTRODUCTION}

Although there are many models of language comprehension, all agree that information about words must be retrieved from long-term memory and that these words must be combined and integrated with other sources of information (Kempen \& Harbusch, 2002; Sag \& Wasow, 1999; Marslen-Wilson, 1987). Many recent accounts of this process are lexicalist in nature, in that the relevant properties of words for language (e.g., phonological, semantic, syntactic) are specified at a lexical level, and the process of building comprehension involves combining these various properties through some sort of combinatorial operation (e.g., "unify"; Vosse \& Kempen, 2000). Although the selection and unification of a word's semantic and syntactic properties likely occurs in parallel (Jackendoff, 2002), these processes may have different time courses and may be processed in different parts of the brain. The neural basis underlying the distinction between the retrieval of lexical information from long-term memory and subse-

\footnotetext{
${ }^{1}$ Max Planck Institute for Psycholinguistics, Nijmegen, The Netherlands, ${ }^{2}$ Radboud University, Nijmegen, The Netherlands

movements were recorded and quantified at two critical sentence regions: a temporarily ambiguous region and a disambiguating region. Results show that stimulation of the IFG led to a modulation of the ambiguity effect (ambiguous-unambiguous) at the disambiguating sentence region in three measures: first fixation durations, total reading times, and regressive eye movements into the region. Both IFG and MTG stimulation modulated the ambiguity effect for total reading times in the temporarily ambiguous sentence region relative to the control group. The current results demonstrate that an offline repetitive TMS protocol can have influences at a different point in time during online processing and provide causal evidence for IFG involvement in unification operations during sentence comprehension. quent integration/unification is specified by the memory unification and control (MUC) framework proposed by Hagoort (2005). In this model, the retrieval of lexical properties is hypothesized to require stored information in posterior temporal regions, whereas unification operations are hypothesized to recruit inferior frontal regions (e.g., the inferior frontal gyrus; IFG). The current work is motivated by these frameworks and seeks to determine whether there is causal evidence for a distinction between retrieval and unification operations during language comprehension through the use of repetitive TMS (rTMS).

Numerous studies have implicated differences between frontal and posterior temporal regions in both syntactic and semantic ambiguity resolution (e.g., Rodd, Johnsrude, \& Davis, 2012; Rodd, Longe, Randall, \& Tyler, 2010; Zempleni, Renken, Hoeks, Hoogduin, \& Stowe, 2007). Important evidence in favor of a distinction between the retrieval and unification of lexical-syntactic information was provided by a series of studies by Snijders and colleagues (Snijders, Petersson, \& Hagoort, 2010; Snijders et al., 2009). This work was based on Vosse and Kempen's (2000) computationally explicit model of syntactic processing, the unification space model. Within this model, every incoming word retrieves one or more lexical frames, which 
specify phonological, semantic, and syntactic properties of the words. In the case of syntax, these lexical frames represent simple syntactic trees specifying the possible structural environment in which the word could occur. Lexical frames enter a unification space one word at a time, where they are maintained as binding operations that incrementally build the syntactic structure of a sentence. Language often contains many ambiguities, such as whether a word is a noun or a verb (word-class ambiguity) or which noun a prepositional phrase modifies (prepositional phrase attachment ambiguity). Within the unification-space model, such ambiguities result in the activation of multiple lexical frames and are resolved through a process of selection by lateral inhibition between lexical nodes as the unification process unfolds over time.

To dissociate retrieval and unification operations, Snijders et al. (2009) generated material containing temporarily word-class ambiguous words with either a noun or verb interpretation (e.g., "run," "rent"). As there is evidence that such words activate both the noun and verb interpretations (Seidenberg, Tanenhaus, Leiman, \& Bienkowski, 1982; Swinney, 1979), word-class ambiguous words were hypothesized to increase demands on retrieval operations relative to matched, unambiguous words, as multiple lexical-syntactic (and semantic) frames would be simultaneously activated. Unification operations in these experiments were manipulated by embedding these words within sentences or random word lists.

On the basis of the MUC framework described above, the authors hypothesized that regions of the posterior, temporal cortex would be sensitive to word-class ambiguity regardless of the sentential context. Sentences necessarily elicit unification/integration operations; thus, the authors predicted that sentences would lead to greater activation of the IFG relative to random word lists. Importantly, because ambiguity leads to increased processing difficulty during unification, the authors hypothesized that the IFG should be sensitive to the ambiguity manipulation only in the sentential context. Results of an initial fMRI experiment confirmed that these predictions as a region of the posterior middle temporal gyrus (MTG) showed sensitivity to the ambiguity of the material regardless of the sentential context, and a region of the posterior IFG (pars opercularis) showed sensitivity to ambiguity within the sentential context only (Snijders et al., 2009). Although the largest activation of the IFG observed in this study was slightly posterior to other investigations of semantic and syntactic ambiguities (which often center around pars triangularis; e.g., Rodd et al., 2010; Zempleni et al., 2007; Rodd, Davis, \& Johnsrude, 2005), an ROI analysis seeded in pars triangularis revealed the same pattern whereby ambiguity effects were only observed in the sentential context. These differences in activation, however, are broadly consistent with the functional organization of the hypothesized within the MUC framework, in which syntactic unification operations are posterior to semantic operations in the IFG (Hagoort, 2005).
In order for the unification operation to function, information must be (1) actively maintained to bind disparate elements, (2) selected if multiple frames are activated, and (3) manipulated such that lexical frames can be combined to form a structural interpretation. Part of the reason that the IFG has been hypothesized as crucial for unification operations is that it demonstrates all of these properties: Neurons in the PFC show the capacity for sustained activation after stimuli have been removed from the environment (e.g., Funahashi, Bruce, \& Golman-Rakic, 1989; Fuster \& Alexander, 1971); it is activated when information must be selected from a set of competing alternatives (e.g., Thompson-Schill, D'Esposito, \& Kan, 1999) and is involved when information must be manipulated in STM (e.g., Miller \& Cohen, 2001). Furthermore, many models of STM, a requisite ability for language comprehension, posit that interactions between frontal and posterior brain areas underlie the active maintenance of domainspecific information (e.g., Miller \& Cohen, 2001; Fuster, 1995). The process of comprehending language occurs over time, and unification operations necessarily require the maintenance of previously encountered words as new words are encountered. Thus, in addition to their individual roles in unification and retrieval, the IFG and MTG should show evidence of being dynamically coupled during language comprehension, and this coupling in turn should be sensitive to the degree of comprehension difficulty. A follow-up study utilizing the same paradigm and materials as Snijders et al. (2009) provided important evidence of this coupling, as the posterior IFG and posterior MTG identified in the initial study showed functional connectivity via a third site in the MTG located superior to the originally identified MTG region (Snijders et al., 2010).

In the case of syntactic information and its unification, there is mounting evidence to suggest a dissociation between the IFG and MTG, with the former subserving unification operations and the latter subserving the retrieval of lexical-syntactic information (e.g., Rodd et al., 2010). Although we would expect that the word-class ambiguity would also create a temporarily semantic ambiguity, the emphasis here on lexical-syntactic retrieval is based on two sets of results. First, in the original study by Snijders et al. (2009), inclusion of a semantic covariate capturing the number of semantic alternatives induced by the ambiguity did not reveal activation in the IFG, nor did it change the regions activated by the word-class ambiguity. Second, converging evidence from structural priming in language production has implicated similar regions of the MTG and IFG (Segaert, Menenti, Weber, Petersson, \& Hagoort, 2012). It is important to note, however, that comprehension requires the dynamic coupling of the IFG and MTG (see Tyler \& Marslen-Wilson, 2008). Given the slow time course of fMRI, there remain a number of open questions about whether the frontal and temporal activation observed in sentential contexts reflects the simultaneous activation of multiple lexical frames or the selection and resolution of the competition between these frames. With 
regard to the latter account, a recent event-related fMRI study found evidence in favor of the IFG involvement during both the initial detection and subsequent resolution of semantic ambiguity, whereas MTG sensitivity to ambiguity only occurred during the later stages of ambiguity resolution (Rodd et al., 2012).

The goal of this study was to address both the causal necessity of the MTG and IFG in sentence comprehension and the timing of their involvement during ambiguity resolution. Causal inference was made by modulating brain activity in these regions with an offline rTMS protocol (continuous theta-burst stimulation [cTBS]; Huang, Edwards, Rounis, Bhatia, \& Rothwell, 2005). To focus on the time course of the ambiguity resolution process, we measured people's eye movements during sentence reading, which simultaneously provide a sensitive measure of initial processing difficulties within particular sentence regions (e.g., initial reading times) and overall processing difficulties that might be associated with selection and unification (e.g., total reading times). We targeted the same IFG and MTG regions previously identified as subserving unification and lexical syntactic retrieval, and we utilized the same word-class (i.e., noun/verb) ambiguous sentence materials and matched controls as Snijders et al. (2009).

Previous eye-tracking studies using temporary lexicalsemantic ambiguities (e.g., bank, port) have revealed early sensitivity upon encountering the ambiguity when the likelihood of each interpretation is equibiased and the context preceding the ambiguity is neutral (e.g., Duffy, Morris, \& Rayner, 1988; see Rayner, 1998, for a review). In cases where the ambiguous word is biased, initial reading times often do not show sensitivity to the ambiguity unless preceding context biases the subordinate meaning. Although the word-class ambiguous words in the current study contained a range of biases toward a noun or verb reading, the average of these biases across the sentences was balanced overall (see Snijders et al., 2009). We reasoned that, if the activation of the MTG reflects the initial retrieval of multiple lexical frames for ambiguous materials, then stimulation of this brain area might modulate initial reading measures within this temporarily ambiguous sentence region (e.g., first fixation durations and eye movements into and out of a region before advancing in the sentence). This ambiguity is resolved toward either the noun or verb reading by virtue of being integrated with later sentence material in a disambiguating sentence region. Hence, we hypothesized that if the IFG is critical to unification operations, then stimulation of this brain area should initially affect processing at the disambiguating portion of the sentence. The process of unification necessarily involves network interaction and the selection of the appropriate interpretation given the sentence context. If activation of the MTG or IFG reflects this selection, or subsequent resolution of the competition between the noun or verb interpretation, then we would expect to see influences of stimulation of these brain areas in reading measures that capture overall reading patterns (e.g., total reading time and total number of fixations).

\section{METHODS}

\section{Participants}

Two groups of participants were included in the study: a TMS group and a control group. For the TMS group, 23 native speakers of Dutch (16 women; mean age $=$ 23.4 years, $S D=2.3$ years) were recruited via the Donders Institute database and through an advertisement placed inside the Donders Institute. All had normal or correctedto-normal vision. The study was approved by the local ethics committee (Commisee Mensgebonden Onderzoek region Arnhem-Nijmegen). All participants were prescreened for contraindications of TMS, were informed of the experimental procedure, and were provided written informed consent according to the guidelines of the local ethics committee. Three people withdrew from the study after the initial, introductory TMS session, leaving a total sample of 20 participants who participated in all sessions.

An age- and education-matched control group was drawn from the same population as the TMS group and was composed of 20 native speakers of Dutch (15 women; mean age $=24.6$ years, $S D=3.5$ years), recruited via the Max Planck Institute Participant Database. All had normal or corrected-to-normal vision and were given informed consent before participation. The use of separate participants as a control group was unavoidable in the present context given the number of controlled, experimental stimuli (see below).

\section{Stimuli}

The experimental sentences were composed of 60 of the original 80 sentences presented in Snijders et al. (2009). The sentences contained a temporarily word-class ambiguous word that could either be resolved as a noun or a verb depending on the sentence context. The original material, although balanced overall, contained some sentences that were relatively more biased toward a noun or verb reading. The sentences selected in this study were chosen to minimize this difference, while providing enough material to counterbalance across four lists. Unlike the original stimuli in which the ambiguity was resolved immediately after the ambiguous word, in the current study, the sentences were modified by inserting a few words between the ambiguous and disambiguating words (see Table 1 for an example). Each of the 60 ambiguous stimuli appeared in either a noun or a verb version (120 sentences in total) and were matched with 60 nonambiguous control sentences that could either have a noun or a verb reading (120 sentences in total). This yielded 240 experimental sentences, which were divided among four experimental lists, containing equal numbers of ambiguous and unambiguous sentences with both 
Table 1. Example Dutch Sentences with and without Word-class Ambiguous Words

\begin{tabular}{|c|c|}
\hline \multicolumn{2}{|c|}{ Ambiguous (Noun Reading) } \\
\hline Dutch & $\begin{array}{l}\text { Je kunt bakken en ook manden fruit } \\
\text { kopen op de markt. }\end{array}$ \\
\hline English (literal) & $\begin{array}{l}\text { You can boxes and also baskets of fruit } \\
\text { buy at the market. }\end{array}$ \\
\hline English & $\begin{array}{l}\text { You can buy boxes and baskets of fruit } \\
\text { at the market. }\end{array}$ \\
\hline
\end{tabular}

Unambiguous (Noun Reading)

Dutch Je kunt dozen en ook manden fruit kopen op de market.

English (literal) You can boxes and also baskets of fruit buy at the market.

English You can buy boxes and baskets of fruit at the market.

\section{Ambiguous (Verb Reading)}

Dutch Je kunt bakken en ook frituren door olie op een hoog vuur te verwarmen.

English (literal)

You can bake and also fry with oil on a high flame to warm.

\section{Unambiguous (Verb Reading)}

Dutch

English (literal)

Je kunt braden en ook frituren door olie op een hoog vuur to verwarmen.

You can roast and also fry with oil on a high flame to warm.

Italic font indicate the ambiguous word, underlined font indicates the temporarily ambiguous sentence region, $\overline{\text { and bold }}$ font indicates the disambiguating sentence region.

noun and verb readings ( 15 sentences in each combination of conditions; e.g., ambiguous-noun, unambiguous-verb, etc.). TMS participants were randomly assigned to each list such that, in one experimental session, they were exposed to an ambiguous version of a sentence and, on a subsequent session (at least 1 week apart; mean $=$ 1.3 weeks, $S D=0.6$ weeks), the unambiguous version of the same sentence. Control participants saw only one version of each list.

In addition to the 60 experimental sentences, 200 filler sentences were generated, 100 of which were presented in each experimental session. Filler sentences were included so that participants would not become aware of the ambiguity manipulation of the experimental sentences. Filler sentences were composed of 80 sentences containing object- or subject-relative clauses (e.g., The boy who the girl kissed/kissed the girl was very happy), 40 passive sentences (e.g., The scarves were worn by an old cello player), 40 sentences containing a sentential complement (e.g., The girl thought that people with beards are scary), and 40 sentences containing a sentential complement with embedded clauses (e.g., The pilots thought that runways with ice are dangerous).

\section{Procedure}

\section{Experimental Procedure}

TMS group. Participants in the study underwent three sessions, each separated by at least 1 week. In the first session, participants were given informed consent and introduced to TMS beginning with single pulse stimulation to establish motor threshold (described below). Following motor threshold, participants were introduced to the stimulation of the two brain areas targeted in this study: MTG and IFG. In the initial introduction of TMS, we did not yet have anatomical scans for participants; thus, the approximate locations of the IFG and MTG were estimated based on the relative scalp position from the vertex. Introductory stimulation began with single pulse stimulation at $80 \%$ of the active motor threshold, followed by $2 \mathrm{sec}$, then $5 \mathrm{sec}$, and then $10 \mathrm{sec}$ of cTBS (described below). This introduction was designed to allow participants to feel the peripheral effects of cTBS (e.g., muscle twitching) to decide if they wanted to participate in the study. If participants chose to participate and did not yet have a high-resolution anatomical MRI, they were then scanned. MRI images were acquired on a 1.5-T scanner (Avanto, Siemens Medical Systems, Erlangen, Germany). Highresolution, T1-weighted anatomical images were acquired using an MP-RAGE sequence (repetition time $=2250 \mathrm{msec}$, echo time $=2.95 \mathrm{msec}, 176$ sagittal slices, voxel size $=$ $1.0 \times 1.0 \times 1.0 \mathrm{~mm}$, field of view $=256 \mathrm{~mm}$ ). MRI scans were then used to guide subsequent TMS stimulation (see below).

On the two subsequent experimental sessions (performed at least 1 week apart), participant eye movements were recorded after cTBS stimulation to each brain area of interest, the order of which was counterbalanced across participants and sessions. Before cTBS, participants were aligned with a head-mounted eye-tracking system (Eyelink II, SR Research Ltd., Mississuaga, Ontario, Canada) and given 10 practice sentences while their eye movements were measured. Each sentence was presented until the participants pressed a mouse button to advance and was followed by a yes/no comprehension question, answered with the left or right mouse button press. Following eyetracker alignment and practice, participants were then aligned with the neuronavigation system (described below), and single pulses of TMS were applied at $80 \%$ of the active motor threshold so participants could be reintroduced to TMS. Following reintroduction, cTBS was applied for $40 \mathrm{sec}$, at which point participants were brought back to the eye tracker and realigned, and the sentence reading began. The total amount of time between stimulation and beginning sentence reading was $5 \mathrm{~min}$. 
Participants then read 160 sentences, and every 20 sentences, there was a chance to recalibrate the eye-tracking equipment if necessary. On average, participants spent $22 \mathrm{~min}(S D=3.4 \mathrm{~min})$ reading sentences.

Control group. Control participants read the same sentences as the TMS participants using the same eye-tracking equipment, but no TMS was applied. The control group served as a baseline and only performed the reading task once. Although this created a situation where TMS participants saw variants on the stimuli more than once, piloting of the experiment revealed stable patterns when the stimuli were read more than once.

\section{TMS Procedure}

TMS was applied using a biphasic Magstim Super Rapid Stimulator (Magstim Company Ltd., Whitland, United Kingdom), using a figure-of-eight coil with a diameter of $70 \mathrm{~mm}$.

Motor threshold. Resting and active motor threshold were attained for each participant. First, a swim cap was placed on the participant's head, and a $5 \times 5 \mathrm{~cm}$ grid was drawn $5 \mathrm{~cm}$ anterior and to the left of the vertex. Participants rested their right hands on a pillow, and electrodes were used to measure motor-evoked potentials (MEPs) in the first dorsal interosseous (FDI) muscle of the right hand using a standard electromyogram recording procedure. The stimulator coil was first placed in the middle of the grid, and the intensity was increased until an MEP was evoked. Following this, the coil was then systematically moved around the $5 \times 5$ grid until the largest MEP was evoked. Stimulator intensity was then decreased, and the resting motor threshold was defined as the minimum intensity needed to elicit reproducible (5 of 10) MEPS in the FDI (minimum peak-to-peak amplitude of $50 \mu \mathrm{V}$ ). During the acquisition of active motor threshold, participants were instructed to maintain FDI muscle contraction at $15 \%$ of their individual maximum voluntary contraction, a target that was visible to participants based on a display of the EMG recordings of their FDI. Stimulator intensity was then lowered, and the active motor threshold was defined as the lowest TMS output needed to evoke a reproducible MEP ( 5 of 10 pulses) in the FDI with a minimum peak-to-peak amplitude of $200 \mu \mathrm{V}$. Mean active motor threshold was $41 \%(S D=5.4 \%)$ of the stimulator output.

cTBS. CTBS was applied to two brain areas based on the group activations in Snijders et al. (2009): MTG (MNI coordinates: $x=-52, y=-50, z=-8$ ) and IFG $(x=-44, y=0, z=22)$. During cTBS, a burst of three $50-\mathrm{Hz}$ pulses are repeated every $200 \mathrm{msec}$, for a total of 600 pulses (40 sec; see Huang et al., 2005, for details). Stimulation was applied at $80 \%$ of each participant's active motor threshold (mean stimulation $=$ $33 \%, S D=4.4 \%$ ).

The two target sites of stimulation, the left MTG and IFG, were localized with neuronavigation. Target brain areas were overlaid onto the participant's anatomical scan by placing a 6-mm sphere around the MNI coordinates for the MTG and IFG. To put these coordinates into participant space, we used the inverse parameters obtained from spatially normalizing the T1 images to the standardized MNI space in SPM5 (www.fil.ion.ucl.ac.uk/spm). To position the TMS coil on the scalp locations overlapping the target sites, the high-resolution T1 scans for each participant were loaded into BrainSight (Rogue Research Inc., Montreal, Quebec, Canada). The TMS coil could then be positioned on the participant's scalp as close to the target region as possible. The coil was placed at a $45^{\circ}$ angle from horizontal in the superior-inferior plane, and in instances where this created discomfort for the participant, this angle was adjusted while maintaining coil placement over the two target brain areas. It is important to note that the IFG coordinates utilized here were sometimes localized on the border of the opercular portion of IFG and the ventral premotor cortex. In instances where the IFG ROI was ambiguous between the two, stimulation was moved toward the anterior portion of the $6-\mathrm{mm}$ sphere so that pars opercularis was targeted.

\section{Data Analysis}

\section{Eye-tracking Measures}

Given the predictions we had about the potential roles of the MTG and IFG during sentence processing, analyses were focused on online measures of eye-tracking behavior. As such, we measured two standard sets of measures that capture online eye-movement behavior during reading (see Rayner, 1998). The first set of measures captures reading durations and included first fixation duration and total reading time for the given sentence regions. First fixation duration captures the total amount of time first spent on fixating a sentence region.

The second set of measures involved eye movements. Here, we utilized three measures: regressive eye movements into a region from an earlier sentence region before advancing ahead in sentence reading; regressive eye movements out of a sentence region to an earlier sentence region before advancing ahead in reading; and the total number of fixations to a given sentence region. Such eye movements capture the active process of reading and should thus be sensitive in resolving the temporary ambiguities as people move their eyes to earlier and later sentence regions.

\section{Data Cleaning}

Before data analysis, all eye movements were manually corrected for drift during a trial. Following this, a standard 
four-stage procedure for cleaning eye movement was conducted using EyeLink Data Viewer (SR Research, Ontario, Canada). To correct for blink-related artifacts, any fixation less than 80 msec was combined with longer fixation if it fell within $0.5^{\circ}$ of visual angle; in a second stage, these parameters were set to $40 \mathrm{msec}$ and $1.25^{\circ}$ of visual angle. In a third stage, if an interest area included three successive fixations less than $140 \mathrm{msec}$, these were combined into one fixation. Finally, fixations less than $140 \mathrm{msec}$ and greater than 800 msec were excluded.

Comprehension questions were not designed to focus on particular parts of the sentence but rather on the extraction of overall meaning. Accuracy was, on average, very high (>94\% overall) and did not show differences between TMS conditions. All analyses below were conducted on correct responses, resulting in the exclusion of $5.3 \%$ of the data.

\section{Data Analysis}

The focus of the current investigation was the influence of TMS to two brain areas, the IFG and MTG, each hypothesized to serve different roles during sentence processing. The measures of eye tracking capture this online processing, and analyses were focused on two sentence regions where the involvement of the MTG and IFG should have been maximal: a temporarily ambiguous region that included the word-class ambiguous word and a disambiguating region where either a noun or a verb was provided that led to the correct noun/verb reading of the ambiguous word. Each of these regions was composed of three words, a critical word and two subsequent words, which controlled for spillover effects (see Table 1).

Participants and items were counterbalanced on both noun or verb reading and the order in which the different brain areas were stimulated. As such, we collapsed across these two variables in the analyses below. Two sets of analyses were conducted at each sentence region (temporarily ambiguous and disambiguating) for each measure of reading behavior. The first analysis compared the two sentence types (ambiguous/unambiguous) for each TMS conditions (IFG and MTG) against the control group. The comparison of TMS groups with control was between participants and within items, whereas sentence type was within participants and items. A second analysis directly compared the two TMS groups composed of a 2 (TMS Condition: MTG/IFG) × 2 (Sentence Type: ambiguous/unambiguous) design that was fully crossed within participants and items.

Data analyses were conducted using linear mixed effects models with the lme 4 package (Bates, 2005) in $\mathrm{R}$ version 2.15.2 (R Core Team, 2012), which allowed us to simultaneously assess random effects of both participants and items within the same analysis (Baayen, Davidson, \& Bates, 2008). Analyses included fixed effects of the two factors in the study and their interactions as well as random in- tercepts for participants and items. Any significant interactions were followed up with comparable mixed effects models and are reported in text.

In the initial analysis, TMS condition was contrast coded using the control group as the reference. Thus, both IFG and MTG stimulations were compared directly with the control group to assess whether there was any effect of TMS to these regions relative to a group that received no TMS. Importantly, if any differences observed between the groups were simply a matter of differences in exposure to the sentence material, we would expect this to come out in the direct comparison between each TMS condition with the control group. Sentence type was mean-centered before analysis, with a code of -1 corresponding to ambiguous and +1 corresponding to unambiguous sentences. In a second analysis, IFG and MTG stimulations were directly compared against each other. For the analysis comparing IFG and MTG stimulation, the IFG and ambiguous sentences were given a code of -1 and MTG and unambiguous sentences were given a code of +1 . Negative numbers for main effects thus correspond to longer reading times or more fixations for those items coded with -1 .

A Poisson linking function was used for the fixation measures as these correspond to count data (Barr, 2008). Statistical significance for the reading times was assessed using 10000 Markov chain Monte Carlo simulations in the pvals.fnc function of the languageR package (Baayen, 2008), and using $p$ values provided for the logistic and Poisson regressions in the case of eye movements.

\section{RESULTS}

\section{Looking Times}

We begin the analysis by focusing the amount of time people spent reading the two critical sentence regions. Figure 1 contains the mean looking times for each TMS condition, sentence type, and sentence region, and Table 2 shows the results of the mixed effects models for each of the looking time measures described below. For the analyses comparing IFG/MTG stimulation with the control group, the intercept corresponds to the mean looking times for the control group.

\section{First Fixation Duration}

First fixation duration captures the amount of time participants first spent reading each sentence region (see Table 2 and Figure 1A). Results of the analysis between the control group and the two TMS groups revealed no significant effects at the temporarily ambiguous sentence region. At the disambiguating region, however, there was a significant interaction between IFG versus control and sentence type, indicating that the magnitude of the difference between ambiguous and unambiguous sentences (hereafter the ambiguity effect) 


\section{$\square$ Ambiguous $\square$ Unambiguous}

\section{A First Fixation Duration}

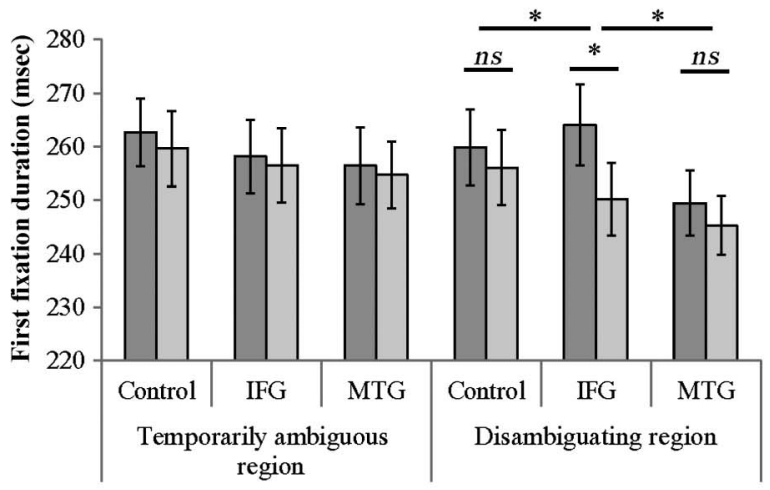

\section{B Total Reading Time}

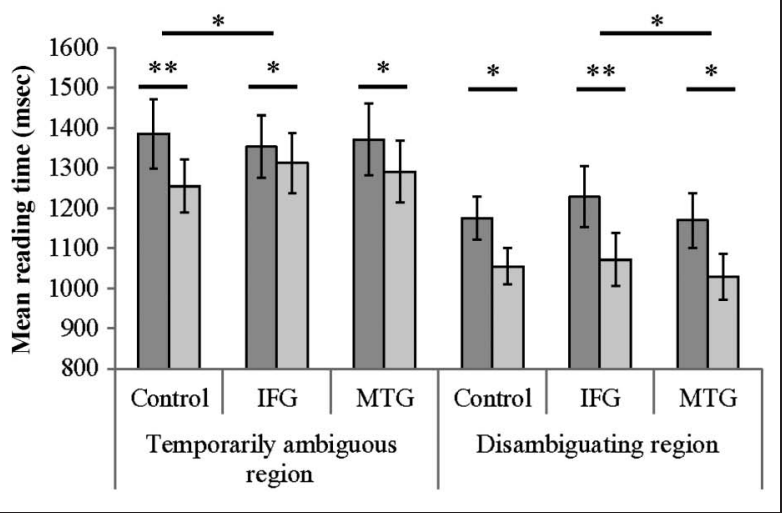

Figure 1. Mean looking times for each sentence region, TMS, and sentence ambiguity condition. Error bars represent the SEM across participants. (A) Mean of first fixation duration. (B) Mean of total looking time in milliseconds.

differed between the IFG and control groups. Follow-up analyses showed that, although no ambiguity effect was observed for the control group $\left(\mu_{\mathrm{D}}=4.2 \mathrm{msec}\right.$, $S D_{\mathrm{D}}=12.6 \mathrm{msec}, \beta=-2.0, S E=1.68, t=-1.14$, $p>$.2), there was a significant difference for the IFG group $\left(\mu_{\mathrm{D}}=13.5 \mathrm{msec}, S D_{\mathrm{D}}=16.6, \beta=-6.9, S E=1.7, t=-3.98\right.$, $p<.001)$.

Comparison with MTG stimulation directly showed similar patterns, although in this instance, there was a main effect of TMS group, with longer reading times following IFG than MTG stimulation $\left(\mu_{\mathrm{D}}=5.4 \mathrm{msec}, S D_{\mathrm{D}}=\right.$ $15.3 \mathrm{msec}$ ), and also a significant TMS Group $\times$ Sentence Type interaction. Similar to the control group, following MTG stimulation, there was no significant ambiguity effect at the disambiguating region $\left(\mu_{\mathrm{D}}=8.6 \mathrm{msec}, S D_{\mathrm{D}}=\right.$ $15.8 \mathrm{msec}, \beta=-2.0, S E=1.66, t=-1.21, p>.2)$. This pattern of results indicates that group differences are not driving the differences the control group-neither IFG nor MTG stimulation slowed down initial reading times relative to the control group. More importantly, the results demonstrate that stimulation of the IFG influenced the processing of ambiguity where initial reading patterns should be most sensitive to ambiguity (i.e., the disambiguating region).

\section{Total Reading Time}

Total reading time measures the total amount of time participants spent in each sentence region and is thus a more coarse measure of online processing than first fixation durations. Still, the measurement of total reading time permits an examination of whether participants were sensitive to sentence ambiguity overall. Furthermore, total reading times are an indication of the overall process of disambiguation, which ultimately requires selection of one syntactic interpretation.

Results of the analysis with the control group (Table 2 and Figure 1B) revealed significant main effects of sentence type for both the temporarily ambiguous and disambiguating regions. As evidenced by the direction of the regression coefficients (ambiguous items were coded as -1 ), participants showed the expected ambiguity effect, with longer reading times for ambiguous than unambiguous sentences $\left(\mu_{\mathrm{D}}=111 \mathrm{msec}, S D_{\mathrm{D}}=194 \mathrm{msec}\right)$. In addition to this main effect, there was also an IFG versus Control $\times$ Sentence Type interaction at the temporarily ambiguous region as well as a nearly significant MTG versus Control $\times$ Sentence Type interaction $(p=.06)$, demonstrating that the magnitude of the ambiguity effect varied between these two groups. Follow-up analyses showed that the interaction was driven by the fact that the ambiguity effect was larger for the control group $\left(\mu_{\mathrm{D}}=133 \mathrm{msec}, S D_{\mathrm{D}}=234 \mathrm{msec}, \beta=-65, S E=9.7, t=\right.$ $-6.72, p<.001)$ relative to the IFG $\left(\mu_{\mathrm{D}}=45 \mathrm{msec}, S D_{\mathrm{D}}=\right.$ 214 msec, $\beta=-27, S E=9.4, t=-2.91, p<.01)$ and MTG groups $\left(\mu_{\mathrm{D}}=58 \mathrm{msec}, S D_{\mathrm{D}}=214 \mathrm{msec}, \beta=-40\right.$, $S E=9.7, t=-4.14, p<.001)$. These latter two groups did not differ. Examination of Figure $1 \mathrm{~B}$ reveals that the difference in the ambiguity effect was driven primarily by TMS stimulation to the IFG and MTG slowing reading for unambiguous sentences. Finally, the direct comparison between IFG and MTG stimulation showed only a main effect of TMS condition at the disambiguating region. As with the first fixation duration, people were overall slower at the disambiguating region following IFG relative to MTG stimulation $\left(\mu_{\mathrm{D}}=14.1 \mathrm{msec}, S D_{\mathrm{D}}=197 \mathrm{msec}\right)$.

\section{Eye Movements}

In addition to measuring looking times, we were also interested in the pattern of fixations that people made during online sentence processing. The results of the mixed effects models are presented in Table 3, and the mean number of each of the different types of fixations is presented in Figure 2. Given the Poisson linking function, regression coefficients correspond to log count proportions and are not directly interpretable in terms of the number of each of the different types of fixations. 
Regressive Eye Movements into a Sentence Region from an Earlier Sentence Region

Results of the mixed effects models with the control group revealed significant main effects of sentence ambiguity at both the temporarily ambiguous and disambiguating sentence regions and also an IFG versus Control $x$ Sentence Type interaction at the ambiguous $(p=.06)$ and disambiguating sentence regions. Examination of Table 3 and Figure 2A shows that the main effect of sentence type was driven by the fact that there were more regressions for ambiguous relative to unambiguous sentences $\left(\mu_{\mathrm{D}}=0.07, S D_{\mathrm{D}}=0.30\right)$. In the case of the ambiguous region, the nearly significant two-way interaction emerged from a larger ambiguity effect for the control group $\left(\mu_{\mathrm{D}}=0.19, S D_{\mathrm{D}}=0.37, \beta=-0.07, S E=0.01\right.$, $z=-5.12, p<.001)$ relative to the IFG group $\left(\mu_{\mathrm{D}}=\right.$ $0.07, S D_{\mathrm{D}}=0.24, \beta=-0.03, S E=0.01, z=-2.17$, $p<.05)$. The reverse was true for the disambiguating region, however, as the magnitude of the ambiguity ef- fect was larger following IFG stimulation $\left(\mu_{\mathrm{D}}=0.24\right.$, $\left.S D_{\mathrm{D}}=0.30, \beta=-0.17, S E=0.02, z=-8.27, p<.0001\right)$ relative to the control group $\left(\mu_{\mathrm{D}}=0.13, S D_{\mathrm{D}}=0.24, \beta=\right.$ $-0.08, S E=0.02, z=-4.14, p<.001)$. Similarly, when comparing IFG and MTG stimulation directly, the interaction between TMS condition and sentence type at the disambiguating region was driven by a larger difference in regressive eye movements into the region following IFG relative to MTG stimulation $\left(\mu_{\mathrm{D}}=0.19, S D_{\mathrm{D}}=0.25, \beta=\right.$ $-0.10, S E=0.02, z=-4.7, p<.001)$.

Thus, the regressive eye movements into a region from an earlier sentence region showed sensitivity to the ambiguity manipulation, demonstrating that participants needed to look into these sentence regions more often to disambiguate them. Importantly, however, these effects were largest in the disambiguating sentence region following IFG stimulation relative to both MTG stimulation and the control group, a result which is consistent with the IFG serving a critical role during unification operations in comprehension.

Table 2. Results of the TMS Condition $\times$ Sentence Ambiguity Mixed Effects Model for Two Different Looking Time Measures and the Two Different Sentence Regions That Were the Focus of This Study

\begin{tabular}{|c|c|c|c|c|c|c|c|c|}
\hline & \multicolumn{4}{|c|}{ Ambiguous Region } & \multicolumn{4}{|c|}{ Disambiguating Region } \\
\hline & $\beta$ & $S E$ & $t$ & $p$ & $\beta$ & $S E$ & $t$ & $p$ \\
\hline \multicolumn{9}{|l|}{ First Fixation Duration } \\
\hline Intercept & 261 & 6.38 & 40.98 & .00 & 259 & 6.43 & 40.23 & .00 \\
\hline IFG vs. control & -3.92 & 8.81 & -0.44 & .67 & -2.46 & 9.00 & -0.27 & .78 \\
\hline MTG vs. control & -6.08 & 8.81 & -0.69 & .49 & -11.31 & 9.00 & -1.26 & .21 \\
\hline Sentence Type & -0.75 & 1.70 & -0.44 & .66 & -2.06 & 1.70 & -1.21 & .23 \\
\hline IFG vs. Control $\times$ Sentence Type & 0.10 & 2.40 & 0.04 & .96 & -4.92 & 2.39 & -2.06 & .04 \\
\hline MTG vs. Control $\times$ Sentence Type & 0.23 & 2.39 & 0.09 & .93 & -0.06 & 2.39 & -0.03 & .97 \\
\hline TMS region (IFG vs. MTG) & -1.09 & 1.18 & -0.92 & .36 & -4.45 & 1.20 & -3.71 & .00 \\
\hline Sentence Type $\times$ TMS Region (IFG vs. MTG) & 0.01 & 1.18 & 0.01 & .99 & 2.39 & 1.20 & 2.00 & .04 \\
\hline \multicolumn{9}{|l|}{ Total Reading Time } \\
\hline Intercept & 1298 & 78.04 & 16.63 & .00 & 1110 & 58.27 & 19.05 & .00 \\
\hline IFG vs. control & 12.24 & 98.89 & 0.12 & .89 & 37.22 & 73.62 & 0.51 & .60 \\
\hline MTG vs. control & 16.13 & 98.88 & 0.16 & .85 & -7.63 & 73.61 & -0.10 & .90 \\
\hline Sentence type & -65.86 & 9.76 & -6.75 & .00 & -59.82 & 9.27 & -6.45 & .00 \\
\hline IFG vs. Control $\times$ Sentence Type & 39.41 & 13.87 & 2.84 & .01 & -19.68 & 13.18 & -1.49 & .13 \\
\hline MTG vs. Control $\times$ Sentence Type & 26.11 & 13.83 & 1.89 & .06 & -9.16 & 13.16 & -0.70 & .47 \\
\hline TMS region (IFG vs. MTG) & 2.23 & 6.92 & 0.32 & .75 & -22.30 & 6.66 & -3.35 & .00 \\
\hline Sentence Type $\times$ TMS Region (IFG vs. MTG) & -6.68 & 6.91 & -0.97 & .33 & 4.94 & 6.65 & 0.74 & .46 \\
\hline
\end{tabular}

The upper portion of each model corresponds to the comparisons of each TMS group with the control (no TMS) group; the bottom portion corresponds to the direct comparison of the IFG and MTG stimulation (within participants).

The intercept corresponds to the mean looking time in msec for the control group. Significant results are indicated in bold font, and nearly significant results $(p<.07)$ are indicated in italics. 
Table 3. Results of the TMS Condition $\times$ Sentence Ambiguity Mixed Effects Model for the Three Different Eye Fixation Measures and the Two Different Sentence Regions That Were the Focus of This Study

\begin{tabular}{|c|c|c|c|c|c|c|c|c|}
\hline & \multicolumn{4}{|c|}{ Ambiguous Region } & \multicolumn{4}{|c|}{ Disambiguating Region } \\
\hline & $\beta$ & $S E$ & $t$ & $p$ & $\beta$ & $S E$ & $t$ & $p$ \\
\hline \multicolumn{9}{|l|}{ Regressive Eye Movements (In) } \\
\hline Intercept & 0.27 & 0.11 & 2.43 & .02 & -0.43 & 0.12 & -3.57 & $<.001$ \\
\hline IFG vs. control & -0.10 & 0.15 & -0.66 & .51 & -0.05 & 0.16 & -0.30 & .77 \\
\hline MTG vs. control & -0.11 & 0.15 & -0.70 & .48 & -0.01 & 0.16 & -0.07 & .94 \\
\hline Sentence type & -0.07 & 0.01 & -5.00 & $<.001$ & -0.08 & 0.02 & -4.22 & $<.001$ \\
\hline IFG vs. Control $\times$ Sentence Type & 0.04 & 0.02 & 1.86 & .06 & -0.09 & 0.03 & -3.13 & $<.002$ \\
\hline MTG vs. Control $\times$ Sentence Type & 0.03 & 0.02 & 1.60 & .11 & -0.02 & 0.03 & -0.68 & .50 \\
\hline TMS region (IFG vs. MTG) & 0.00 & 0.01 & -0.30 & .76 & 0.02 & 0.01 & 1.23 & .22 \\
\hline Sentence Type $\times$ TMS Region (IFG vs. MTG) & 0.00 & 0.01 & -0.19 & .85 & 0.04 & 0.01 & 2.39 & .02 \\
\hline \multicolumn{9}{|l|}{ Regressive Eye Movements (Out) } \\
\hline Intercept & -0.95 & 0.13 & -7.21 & $<.001$ & -0.93 & 0.11 & -8.17 & $<.001$ \\
\hline IFG vs. control & 0.11 & 0.18 & 0.64 & .52 & 0.04 & 0.15 & 0.24 & .81 \\
\hline MTG vs. control & 0.25 & 0.18 & 1.39 & .16 & 0.01 & 0.15 & 0.07 & .95 \\
\hline Sentence type & -0.09 & 0.02 & -3.61 & $<.001$ & -0.12 & 0.03 & -4.51 & $<.001$ \\
\hline IFG vs. Control $\times$ Sentence Type & 0.03 & 0.03 & 1.03 & .30 & 0.00 & 0.04 & 0.05 & .96 \\
\hline MTG vs. Control $\times$ Sentence Type & 0.07 & 0.03 & 2.04 & .04 & 0.05 & 0.04 & 1.25 & .21 \\
\hline TMS region (IFG vs. MTG) & 0.07 & 0.02 & 4.12 & $<.001$ & -0.01 & 0.02 & -0.79 & .43 \\
\hline Sentence Type $\times$ TMS Region (IFG vs. MTG) & 0.02 & 0.02 & 1.02 & .31 & 0.02 & 0.02 & 1.23 & .22 \\
\hline \multicolumn{9}{|l|}{ Total Fixations } \\
\hline Intercept & 1.58 & 0.06 & 27.09 & $<.001$ & 1.45 & 0.05 & 29.52 & $<.001$ \\
\hline IFG vs. control & 0.04 & 0.07 & 0.58 & .56 & 0.05 & 0.06 & 0.84 & .40 \\
\hline MTG vs. control & 0.05 & 0.07 & 0.66 & .51 & 0.04 & 0.06 & 0.59 & .56 \\
\hline Sentence type & -0.05 & 0.01 & -6.32 & $<.001$ & -0.05 & 0.01 & -6.21 & $<.001$ \\
\hline IFG vs. Control $\times$ Sentence Type & 0.03 & 0.01 & 3.20 & $<.002$ & 0.00 & 0.01 & -0.02 & .98 \\
\hline MTG vs. Control $\times$ Sentence Type & 0.02 & 0.01 & 1.71 & .09 & -0.01 & 0.01 & -0.80 & .42 \\
\hline TMS region (IFG vs. MTG) & 0.00 & 0.01 & 0.62 & .54 & -0.01 & 0.01 & -1.32 & .19 \\
\hline Sentence Type $\times$ TMS Region (IFG vs. MTG) & -0.01 & 0.01 & -1.50 & .13 & 0.00 & 0.01 & -0.83 & .40 \\
\hline
\end{tabular}

The upper portion of each model corresponds to the comparisons of each TMS group with the control (no TMS) group; the bottom portion corresponds to the direct comparison of the IFG and MTG stimulation (within participants).

A Poisson linking function was used, hence coefficients correspond to the log sum of each type of fixation. Significant results are indicated in bold font, and nearly significant results $(p<.07)$ are indicated in italics.

Regressive Eye Movements out of a Sentence Region to an Earlier Sentence Region

Similar to the analysis above, regressive eye movements out of a region were also sensitive to the ambiguity of the sentence. Examination of Table 3 and Figure $2 \mathrm{~B}$ show that there were more regressive eye movements for ambiguous than unambiguous sentences $\left(\mu_{\mathrm{D}}=0.04, S D_{\mathrm{D}}=0.20\right)$. In addition, there were significant interactions between MTG versus Control $\times$ Sentence Type indicating that the magnitude of the ambiguity effect varied between the control and MTG stimulation conditions.

Follow-up analyses showed that, although there was a significant ambiguity effect for the control group $\left(\mu_{\mathrm{D}}=0.08\right.$, 
$\left.S D_{\mathrm{D}}=0.11, \beta=-0.08, S E=0.02, z=-3.45, p<.001\right)$, no such difference was observed following MTG stimulation $\left(\mu_{\mathrm{D}}=0.03, S D_{\mathrm{D}}=0.08, \beta=-0.03, S E=0.02, z=-1.12\right.$, $p>.2$ ). Examination of Figure $2 \mathrm{~B}$ reveals that this effect was driven primarily by comparable regressive eye movements out to earlier sentence regions for ambiguous and

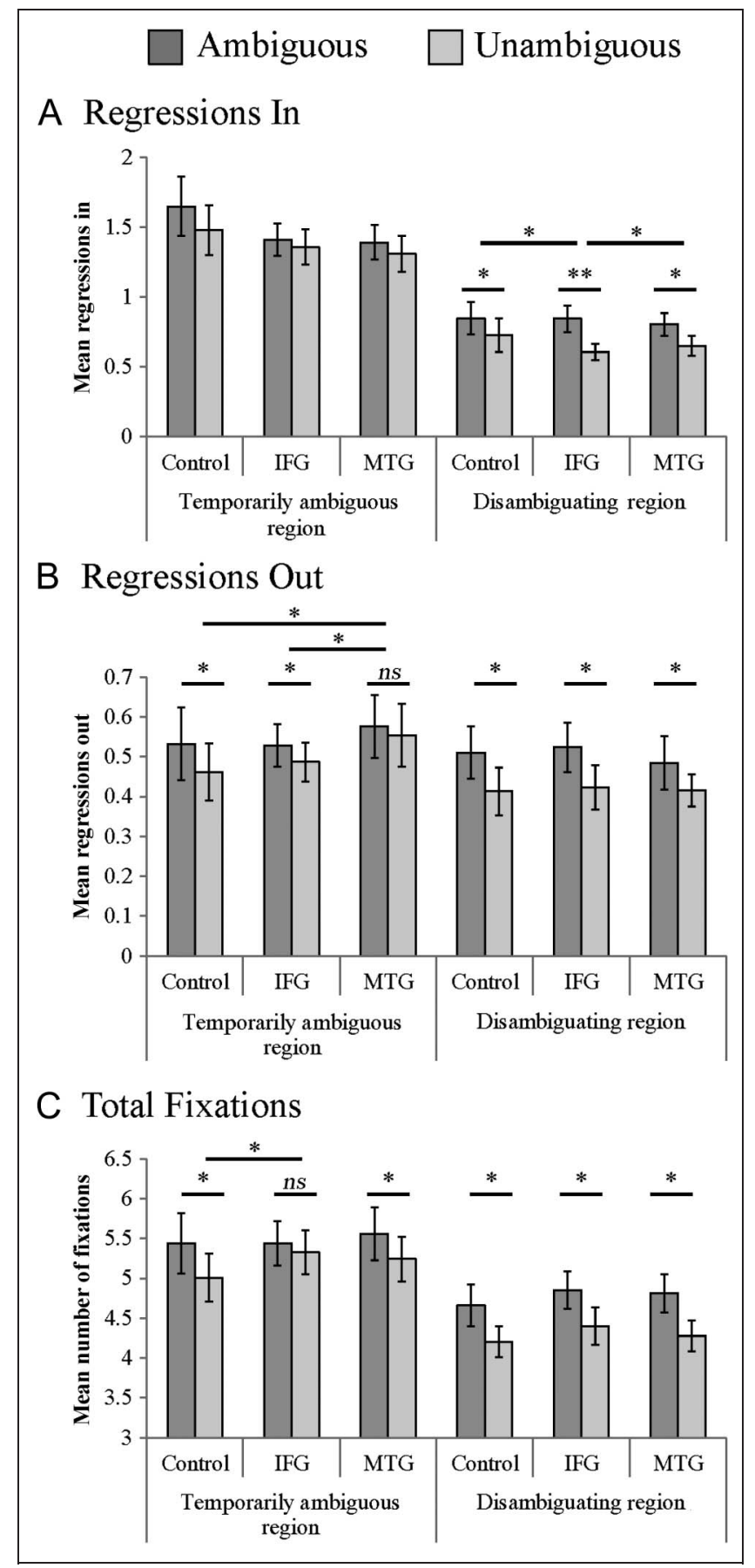

Figure 2. Mean fixation counts for each sentence region, TMS, and sentence ambiguity condition. Error bars represent the SEM across participants. (A) Mean number of regressions into a sentence region from an earlier region before moving on to a later sentence region. (B) Mean number of regressions out to an earlier sentence region before moving to a later sentence region. (C) Mean number of fixations. unambiguous sentences at the temporarily ambiguous region. This effect, in turn, led to the overall main effect of TMS condition when comparing IFG and MTG stimulation at the temporarily ambiguous region, as there were overall more regressive eye movements following MTG than IFG stimulation $\left(\mu_{\mathrm{D}}=0.03, S D_{\mathrm{D}}=0.17\right)$. Given that regressive eye movements out of a region measure looks to earlier sentence regions before moving on in reading, these results demonstrate the people were more likely to restart reading the entire sentence following MTG stimulation relative to IFG stimulation and relative to the control group.

\section{Total Fixations}

Similar to the analysis of total reading time, the analysis of total fixations provides a relatively coarse measure of online sentence processing but does provide an indication of how much processing went on in a given sentence region. Results of the analysis on total fixations revealed main effects of sentence type at both the ambiguous and disambiguating regions as well as an IFG versus Control $\times$ Sentence Type interaction at the ambiguous region (see Table 3; Figure 2C).

Paralleling earlier results, the main effect of sentence type showed that people were sensitive to the ambiguity in the sentences as there were more fixations for ambiguous relative to unambiguous sentences at both the ambiguous $\left(\mu_{\mathrm{D}}=0.28, S D_{\mathrm{D}}=0.80\right)$ and disambiguating $\left(\mu_{\mathrm{D}}=\right.$ $\left.0.48, S D_{\mathrm{D}}=0.70\right)$ sentence regions. The IFG versus Control $\times$ Sentence Type interaction at the temporarily ambiguous region paralleled the results for regressive eye movements into a region as the magnitude of the ambiguity effect was larger for the control group (stimulation: $\mu_{\mathrm{D}}=$ $0.46, S D_{\mathrm{D}}=0.80, \beta=-0.05, S E=0.007, z=-6.31$, $p<.001)$ relative to IFG stimulation, which failed to reach significance $\left(\mu_{\mathrm{D}}=0.12, S D_{\mathrm{D}}=0.86, \beta=-0.01, S E=\right.$ $0.007, z=-1.88, p<.06)$. Thus, participant's eye movement patterns revealed a sensitivity to sentence ambiguity overall. Furthermore, this ambiguity effect was modulated after IFG stimulation in the temporarily ambiguous sentence region, although this was driven primarily by differences in the unambiguous condition.

\section{GENERAL DISCUSSION}

The present investigation was designed to test whether the unification and retrieval of lexical-syntactic information can be dissociated in the brain during sentence comprehension. Using group activation maps from an earlier fMRI study that found such dissociations (Snijders et al., 2009), an offline inhibitory rTMS protocol (cTBS) was used to target two critical nodes of the language network (the IFG and the MTG) hypothesized to serve dissociable roles in the unification operations and lexical-syntactic retrieval (Hagoort, 2005). Eye movements were recorded as people read sentences containing temporary word-class ambiguities, and both reading times and eye movements were 
used to assess performance. Some measures captured initial reading performance (first fixation duration, regressions into and out of a region), and others captured overall reading patterns (total reading time and total number of fixations). Given that there is evidence that both noun and verb interpretations of a word are accessed upon encountering a word-class ambiguity, we predicted that, for the initial measures of reading performance, stimulation of the MTG would affect processing at the temporarily ambiguous sentence region whereas stimulation of the IFG would affect the process of disambiguating the sentence material. Results showed that participants were sensitive to the ambiguity of the sentence material as there were longer reading times and more fixations for ambiguous relative to unambiguous sentences across many measures. More importantly, the results provided clear evidence for a role of the IFG in unification operations in both early and later processing measures. We did not find evidence for a specific role of the MTG during the initial stages of lexicalsyntactic retrieval, but there was some evidence for MTG involvement in the subsequent resolution of the ambiguity.

The overall pattern of reading results revealed sensitivity to the ambiguity manipulation, although this was not present in the initial reading times in the ambiguous sentence regions. Although numerous studies utilizing balanced lexical-semantic ambiguities have found that ambiguity slows reading times (e.g., Duffy et al., 1988), other studies using word-class ambiguities have sometimes failed to find initial differences at ambiguous sentence regions (e.g., Boland \& Blodgett, 2001). Similar to previous studies, however, sensitivity to the ambiguity was present later during sentence reading at the disambiguating sentence region. Importantly, stimulation of the IFG led to a number of changes in reading behavior that are consistent with the claim that the IFG playing a critical role in unification operations.

As predicted, rTMS to the IFG led to a modulation of the ambiguity effect in the initial reading times at the disambiguating sentence region; no such effect was observed either for the control group or after MTG stimulation. A similar effect was also observed for regressive eye movements into the disambiguating sentence region before continuing with sentence reading, with larger ambiguity effects following IFG stimulation. Both of these results are consistent with IFG stimulation affecting the initial sensitivity to ambiguity. Furthermore, the more coarse measures of sentence processing also showed an effect of IFG stimulation at the temporarily ambiguous sentence region, although in this case, the effect of TMS was to reduce the ambiguity effect relative to the control group. The early effects thus demonstrate an increased sensitivity to ambiguity following IFG stimulation at the sentence location that should be most sensitive (i.e., the disambiguating region). The later effects may reflect sustained activation and integration of information within the part of the sentence causing the ambiguity, a process that would help select the appropriate interpretation. This pattern of results is thus broadly consistent with a recent fMRI study demonstrating IFG sensitivity to semantic ambiguity both when the ambiguity was initially encountered and when it was subsequently resolved (Rodd et al., 2012).

The latter pattern of results on the overall sentence processing measures deserves some consideration as IFG stimulation seems to have reduced rather than enhanced the ambiguity effect. In both instances of IFG affecting the temporarily ambiguous region, the reduction in ambiguity effect was driven by influences on the unambiguous rather than the ambiguous sentences. There are a number of possible reasons why this might have occurred. TMS of the IFG could have created more indeterminacy overall, hence participants may have fixated between the temporarily ambiguous and disambiguating sentence regions equally as these two regions correspond to the primary place where thematic role assignment was occurring. Alternatively, it is possible that stimulation of the IFG simply led to less re-reading of earlier sentence constituents overall regardless of the ambiguity. In this instance, people may have simply continued reading the sentence, relying on longer fixations to the disambiguating region to resolve the ambiguity.

A central claim of the MUC framework is that unification operations generally (and IFG involvement specifically) reflect not only integration but also the active maintenance and selection of information stored in posterior brain areas. This interaction has been documented across a number of domains including vision (e.g., Funahashi et al., 1989; Fuster \& Alexander, 1971) and language (Saur et al., 2008). The functional and anatomical connectivity between frontal and posterior brain areas involved in language has been well established (e.g., dorsal and ventral pathways; Glasser \& Rilling, 2008; Catani, Jones, \& Ffytche, 2005). In the present investigation, the dissociation between the initial and overall reading effects after IFG stimulation may be an indication of the dynamic interaction between these frontal and posterior brain areas. More specifically, the initial detection of ambiguity might trigger increased coupling to posterior brain areas to ultimately select one or another meaning. This increased coupling would have two effects: First, it would require active maintenance of multiple possible interpretations before their resolution; second, this active maintenance would then lead to the modulation of reading behavior in earlier sentence regions. Interestingly, these results point to the fact that the exact same TMS stimulation can effect processing at different points in time as a task unfolds. An important area for future research, then, will be to incorporate neural measures of the brain's network dynamics (e.g., EEG, MEG, etc.) to determine how TMS can affect these network interactions.

The current pattern of results is broadly consistent with the IFG involvement in the selection among competing alternatives (e.g., Thompson-Schill, D'Esposito, Aguirre, \& Farah, 1997). Other studies of semantic and syntactic ambiguity resolution have assumed that IFG involvement 
might reflect this early selection mechanism as people commit themselves to one particular sentence interpretation, whereas later IFG and posterior temporal lobe involvement reflect sentence re-analysis (e.g., Rodd et al., 2012; Zempleni et al., 2007). The pattern of results in this and other studies, however, are equally consistent with multiple parses being simultaneously maintained, where the ultimate selection of the correct interpretation occurs as a result of later, disambiguating sentence material. Contrary to some views maintaining early commitment to a single sentence interpretation (e.g., the garden-path theory; Frazier, 1987), numerous studies in sentence processing (see MacDonald, Pearlmutter, \& Seidenberg, 1994) and visual world eye tracking (Tanenhaus, SpiveyKnowlton, Eberhard, \& Sedivy, 1995) have demonstrated that individuals can maintain more than one sentence interpretation simultaneously, utilizing multiple probabilistic sources of information to converge on the appropriate interpretation. In the current study, the word-class ambiguity may have led to the temporary activation of both the noun and verb readings and, thus, two competing representations that needed to be resolved (i.e., selected). We believe that the selection among competing alternatives is one among a larger set of unification operations, which also include maintenance and integration (see Hagoort, 2005). From this perspective, early IFG involvement when there is an ambiguity may reflect the need to maintain multiple potential interpretations simultaneously, whereas subsequent IFG and MTG involvement would reflect the selection of one particular interpretation after integrating multiple probabilistic constraints.

Within the unification space model, for instance, ambiguity leads to the activation and subsequent maintenance of multiple lexical representations; selection emerges as a result of lateral inhibition between the competing lexical elements that are actively maintained (Vosse \& Kempen, 2000). Critically, the process of selection within this model is not driven by a top-down biasing signal from a domaingeneral control network (e.g., in biased competition; Desimone \& Duncan, 1995). Rather, the bias for a particular interpretation emerges from the integration of probabilistic information across the range of linguistic information that is maintained through the IFG (e.g., phonological, semantic, syntactic; Sahin, Pinker, Cash, Schomer, \& Halgren, 2009). From this perspective, the process of unification described here is similar to constraint-satisfaction approaches to language comprehension (e.g., MacDonald et al., 1994; Trueswell \& Tanenhaus, 1994). This is not to say that domain-general control does not exert such an influence over this unification process (e.g., Novick, Trueswell, \& Thompson-Schill, 2005), only that a critical source of biasing information emerges from interactions among the sorts of linguistic (and extralinguistic) information that are maintained within the IFG. In the current investigation, the only means by which the information could have been properly selected is by integrating with the later sentence material that in turn biased the interpre- tation toward the noun or verb reading of the word. The connectivity profile of the IFG and its ability to actively maintain information provides an ideal neural substrate in which information could be integrated such that selection via biased competition could take place.

Given the above-described network dynamics involved in language processing, it is noteworthy that we failed to find more robust effects of MTG stimulation. Although stimulation of the MTG did affect people's processing at the temporarily ambiguous region, with more outside regressions overall, these early effects were not sensitive to the ambiguity of the material. Thus, participants were more likely to re-read the beginning of the sentence after MTG stimulation, but the insensitivity of this effect to the ambiguity manipulation is not consistent with TMS affecting lexical-syntactic retrieval in the MTG. Stimulation of this brain region did, however, show similar effects to IFG stimulation on the total reading times, in this case, reducing the magnitude of the ambiguity effect relative to the control group. Although this result was only marginally significant, it is consistent with a recent fMRI study demonstrating MTG sensitivity to semantic ambiguity resolution only at the point in time when the ambiguity is resolved (Rodd et al., 2012).

One possible reason that MTG stimulation did not show more robust results is that it may simply be more difficult to target the MTG with TMS than the IFG, perhaps because of anatomical variability or the cortical depth at which processing is taking place. A second possibility is that, in balancing the sentence material for noun/verb reading, we may have created a situation in which early processing differences at the ambiguous sentence region would be difficult to observe. Prior behavioral work has shown that sensitivity to the initial processing of an ambiguity in sentence comprehension is more likely to occur either when the material is completely balanced or when the preceding context biases the subordinate interpretation when the material is not balanced (e.g., Duffy et al., 1988; Frazier \& Rayner, 1987). In the current study, disambiguating contextual information was only provided after the ambiguity. Furthermore, although the material was balanced across the sentences, there was a range of bias toward a noun or verb interpretation. It is thus possible that the material in this study may not have been optimized to reveal early effects of MTG stimulation, and in future research, it may be worth considering using other types of ambiguity that have revealed robust effects of lexical constraints (e.g., temporary structural ambiguities; MacDonald et al., 1994; Trueswell, Tanenhaus, \& Garnsey, 1994). Still, the later sensitivity to MTG stimulation is consistent with some role of the MTG during the resolution of the ambiguity and suggests that the earlier fMRI results from Snijders et al. (2009) may, in part, reflect the sustained activation of lexical information.

Numerous studies have now implicated the MTG as critical for retrieving lexical-syntactic information in both comprehension (Rodd et al., 2010; Snijders et al., 2009) 
and production (Menenti, Segaert, \& Hagoort, 2012). Furthermore, activation and stimulation of the MTG has been associated with accessing semantic information (Whitney, Kirk, O'Sullivan, Lambon Ralph, \& Jefferies, 2012; Acheson, Hamidi, Binder, \& Postle, 2011; Binder, Desai, Graves, \& Conant, 2009) and in semantic control (Hoffman, Pobric, Drakesmith, \& Lambon-Ralph, 2012; Whitney et al., 2012). It is noteworthy that many TMS studies that have found specific MTG involvement during semantic processing have either used measures that emphasize the need to integrate semantic information with earlier sentence information (e.g., Franzmeier, Hutton, \& Ferstl, 2012) or the need to select a subordinate meaning (e.g., Hoffman et al., 2012; Whitney et al., 2012). Combined with the present results, these findings provide evidence that at least some of the MTG activation found in fMRI studies may not reflect the initial accessing of lexical-semantic/syntactic information but, rather, the subsequent selection and integration of this information. The posterior MTG is, however, a large region, and it remains plausible that inferior portions of the MTG as well as inferior temporal regions may support the retrieval of lexical information (Snijders et al., 2009; Rodd et al., 2005), where more superior portions are critical for the subsequent integration of this information (see Snijders et al., 2010; Lau, Phillips, \& Poeppel, 2008). It thus remains an important area for future research to use TMS to delineate the nature of the representation and the processing occurring within different regions of the MTG.

In conclusion, the current study provides three important results. First, the study demonstrates that an offline rTMS protocol can show effects that occur at different points in time as processing unfolds. Second, the results are consistent with a role of the MTG in the subsequent selection and integration of lexical-syntactic information during online sentence comprehension. Finally, the present investigation provides evidence supporting a critical role of the IFG both in the maintenance of multiple syntactic interpretations and in the subsequent selection of one of these interpretations. These results, in turn, are consistent with the IFG serving as a critical neural substrate for unification operations during language comprehension.

\section{Acknowledgments}

Funding for this research was supported by the Max Planck Society. We thank Tinneke Snijders for sharing stimuli, Moniek Munneke for technical assistance with TMS, and Tomas Bergvelt and Livia van de Kraats for assistance with experimental testing.

Reprint requests should be sent to Daniel J. Acheson, Max Planck Institute for Psycholinguistics, Box 310, $6500 \mathrm{AH}$ Nijmegen, The Netherlands, or via e-mail: Dan.Acheson@mpi.nl.

\section{REFERENCES}

Acheson, D. J., Hamidi, M., Binder, J. R., \& Postle, B. R. (2011). A common neural substrate for language production and verbal working memory. Journal of Cognitive Neuroscience, 23, 1358-1367.

Baayen, R. H. (2008). Analyzing linguistic data: A practical introduction to statistics using $R$. Cambridge, UK: Cambridge University Press.

Baayen, R. H., Davidson, D. J., \& Bates, D. M. (2008). Mixed-effects modeling with crossed random effects for subjects and items. Journal of Memory and Language, 59, 390-412.

Barr, D. J. (2008). Analyzing "visual world" eyetracking data using multilevel logistic regression. Journal of Memory and Language, 59, 457-474.

Bates, D. M. (2005). Fitting linear mixed models in R. $R$ News, 5, 27-30.

Binder, J. R., Desai, R. H., Graves, W. W., \& Conant, L. L. (2009). Where is the semantic system? A critical review and meta-analysis of 120 functional neuroimaging studies. Cerebral Cortex, 19, 2767-2796.

Boland, J. E., \& Blodgett, A. (2001). Understanding the constraints on syntactic generation: Lexical bias and discourse congruency effects on eye movements. Journal of Memory and Language, 45, 391-411.

Catani, M., Jones, D. K., \& Ffytche, D. H. (2005). Perisylvian language networks of the human brain. Annals of Neurology, 57, 8-16.

Desimone, R., \& Duncan, J. (1995). Neural mechanisms of selective visual attention. Annual Review of Neuroscience, 18, 193-222.

Duffy, S. A., Morris, R. K., \& Rayner, K. (1988). Lexical ambiguity and fixation times in reading. Journal of Memory and Language, 27, 429-446.

Franzmeier, I., Hutton, S. B., \& Ferstl, E. C. (2012). The role of the temporal lobe in contextual sentence integration: A single-pulse transcranial magnetic stimulation study. Cognitive Neuroscience, 2, 1-7.

Frazier, L. (1987). Theories of sentence processing. Cambridge, MA: The MIT Press.

Frazier, L., \& Rayner, K. (1987). Resolution of syntactic category ambiguities: Eye movements in parsing lexically ambiguous sentences. Journal of Memory and Language, 26, 505-526.

Funahashi, S., Bruce, C. J., \& Golman-Rakic, P. S. (1989). Mnemonic coding of visual space in the monkey's dorsolateral prefrontal cortex. Journal of Neurophysiology, 61, 331-349.

Fuster, J. M. (1995). Memory in the cerebral cortex. Cambridge, MA: MIT Press.

Fuster, J. M., \& Alexander, G. E. (1971). Neuron activity related to short-term memory. Science, 173, 652-654.

Glasser, M. F., \& Rilling, J. K. (2008). DTI tractography of the human brain's language pathways. Cerebral Cortex, 18, 2471-2482.

Hagoort, P. (2005). On Broca, brain, and binding: A new framework. Trends in Cognitive Sciences, 9, 416-423.

Hoffman, P., Pobric, G., Drakesmith, M., \& Lambon-Ralph, M. A. (2012). Posterior middle temporal gyrus is involved in verbal and non-verbal semantic cognition: Evidence from rTMS. Aphasiology, 26, 1119-1130.

Huang, Y.-Z., Edwards, M. J., Rounis, E., Bhatia, K. P., \& Rothwell, J. C. (2005). Theta burst stimulation of the human motor cortex. Neuron, 45, 201-206.

Jackendoff, R. (2002). Foundations of language: Brain, meaning, grammar and evolution. Oxford, UK: Oxford University Press.

Kempen, G., \& Harbusch, K. (2002). Performance grammer: A declarative definition. In M. Theune, A. Nijholt, \& H. Hondorp (Eds.), Computational linguistics in the Netherlands 2001 (pp. 148-162). Amsterdam: Rodopi. 
Lau, E., Phillips, C., \& Poeppel, D. (2008). A cortical network for semantics: (De)constructing the N400. Nature Reviews Neuroscience, 9, 920-933.

MacDonald, M. C., Pearlmutter, N. J., \& Seidenberg, M. S. (1994). The lexical nature of syntactic ambiguity resolution. Psychological Review, 101, 676-703.

Marslen-Wilson, W. (1987). Functional parallelism in spoken word-recognition. Cognition, 25, 71-102.

Menenti, L., Segaert, K., \& Hagoort, P. (2012). The neuronal infrastructure of speaking. Brain and Language, 122, 71-80.

Miller, E. K., \& Cohen, J. D. (2001). An integrative theory of prefrontal cortex function. Annual Review of Neuroscience, 24, 167-202.

Novick, J. M., Trueswell, J. C., \& Thompson-Schill, S. L. (2005). Cognitive control and parsing: Reexamining the role of Broca's area in sentence comprehension. Cognitive Affective \& Behavioral Neuroscience, 5, 263-281.

R Core Team. (2012). R: A language and environment for statistical computing (version 2.15.2). Vienna, Austria: $\mathrm{R}$ Core Team.

Rayner, K. (1998). Eye movements in reading and information processing: 20 Years of research. Psychological Bulletin, 124, 372-422.

Rodd, J. M., Davis, M. H., \& Johnsrude, I. S. (2005). The neural mechanisms of speech comprehension: fMRI studies of semantic ambiguity. Cerebral Cortex, 15 , 1261-1269.

Rodd, J. M., Johnsrude, I. S., \& Davis, M. H. (2012). Dissociating frontotemporal contributions to semantic ambiguity resolution in spoken sentences. Cerebral Cortex, 22, 1761-1773.

Rodd, J. M., Longe, O. A., Randall, B., \& Tyler, L. K. (2010) The functional organisation of the fronto-temporal language system: Evidence from syntactic and semantic ambiguity Neuropsychologia, 48, 1324-1335.

Sag, I. A., \& Wasow, T. (1999). Syntactic theory: A formal introduction. Palo Alto, CA: CSLI Publications.

Sahin, N. T., Pinker, S., Cash, S. S., Schomer, D., \& Halgren, E. (2009). Sequential processing of lexical, grammatical and phonological information within Broca's area. Science, 326, 445-449.

Saur, D., Kreher, B. W., Schnell, S., Kummerer, D., Kellmeyer, P., Vry, M. S., et al. (2008). Ventral and dorsal pathways for language. Proceedings of the National Academy of Sciences, U.S.A., 105, 18035-18040.

Segaert, K., Menenti, L., Weber, K., Petersson, K. M., \& Hagoort, P. (2012). Shared syntax in language production and language comprehension-An fMRI study. Cerebral Cortex, 22, 1622-1670.

Seidenberg, M. S., Tanenhaus, M. K., Leiman, J. M., \& Bienkowski, M. (1982). Automatic access of the meanings of ambiguous words in context-Some limitations of knowledge-based processing. Cognitive Psychology, 14 , 489-537.

Snijders, T. M., Petersson, K. M., \& Hagoort, P. (2010). Effective connectivity of cortical and subcortical regions during unification of sentence structure. Neuroimage, 52, 1633-1644.

Snijders, T. M., Vosse, T., Kempen, G., Van Berkum, J. J., Petersson, K. M., \& Hagoort, P. (2009). Retrieval and unification of syntactic structure in sentence comprehension: An fMRI study using word-category ambiguity. Cerebral Cortex, 19, 1493-1503.

Swinney, D. A. (1979). Lexical access during sentence comprehension-(Re)consideration of context effects. Journal of Verbal Learning and Verbal Behavior, 18, 645-659.

Tanenhaus, M. K., Spivey-Knowlton, M. J., Eberhard, K. M., \& Sedivy, J. C. (1995). Integration of visual and linguistic information in spoken language comprehension. Science, 268, 1632-1634.

Thompson-Schill, S. L., D’Esposito, M., Aguirre, G. K., \& Farah, M. J. (1997). Role of the left prefrontal cortex in retrieval of semantic knowledge: A re-evaluation. Proceedings of the National Academy of Sciences, U.S.A., 94, 14792-14797.

Thompson-Schill, S. L., D’Esposito, M., \& Kan, I. P. (1999). Effects of repetition and competition activity from prefrontal cortex in executive control of memory retrieval. Neuron, 23, 513-522.

Trueswell, J., \& Tanenhaus, M. (1994). Toward a lexical framework of constraint-based syntactic ambiguity resolution. In C. Clifton, L. Frazier, \& K. Rayner (Eds.) Perspectives on sentence processing (pp. 155-179). Hillsdale, NJ: Lawrence Erlbaum.

Trueswell, J. C., Tanenhaus, M. K., \& Garnsey, S. M. (1994). Semantic influences on parsing: Use of thematic role information in syntactic ambiguity resolution. Journal of Memory and Language, 33, 285-318.

Tyler, L. K., \& Marslen-Wilson, W. (2008). Fronto-temporal brain systems supporting spoken language comprehension. Philosophical Transactions of the Royal Society of London, Series B, Biological Sciences, 363, 1037-1054.

Vosse, T., \& Kempen, G. (2000). Syntactic structure assembly in human parsing: A computational model based on competitive inhibition and a lexicalist grammar. Cognition, 75, 105-143.

Whitney, C., Kirk, M., O’Sullivan, J., Lambon Ralph, M. A., \& Jefferies, E. (2012). The neural organization of semantic control: TMS evidence for a distributed network in left inferior frontal and posterior middle temporal gyrus. Cerebral Cortex, 21, 1066-1075.

Zempleni, M. Z., Renken, R., Hoeks, J. C., Hoogduin, J. M., \& Stowe, L. A. (2007). Semantic ambiguity processing in sentence context: Evidence from event-related fMRI. Neuroimage, 34, 1270-1279. 